\title{
Mangled finger salvage using cross-finger revascularization
}

\author{
Song Chen ${ }^{1}$, Gen Wen ${ }^{1}$, Liang Cheng ${ }^{1 *}$ and Yimin Chai ${ }^{2}$
}

\begin{abstract}
Background: Mangled finger with impaired arteria digitalis communis remains to be a challenge for replantation surgery due to the limited amount of tissue to work with.

Methods: Out of 554 hands with total finger amputations treated by replantation of finger/fingers from July 2012 to June 2018, there were 7 cases of damaged arteria digitalis communis, all of which were replanted by anastomosing distal adjacent radial/ulnar digital artery to distal end of ulnar/radial digital artery of amputation finger, and 2 veins were anastomosed for each finger. A skin pedicle was made by suturing both dorsal and palmar skin of adjacent fingers, and detachment was performed 4 weeks postoperatively.

Results: The survival rate was $100 \%$. Mean total active motion was $191.4^{\circ}$ (ranging from 170 to $220^{\circ}$ ). Mean 2-point discrimination was $8 \mathrm{~mm}$ static (ranging from 6 to $11 \mathrm{~mm}$ ), and mean grip strength was $35.3 \mathrm{~kg}$ (range, 29 to $40 \mathrm{~kg}$ ).

Conclusions: Based on our experience, cross-finger revascularization is an effective and safe alternative for mangled finger salvage when arteria digitalis communis is damaged, and good functional prognosis can be expected.
\end{abstract}

Keywords: Replantation, Cross-finger revascularization, Microsurgery

\section{Background}

Komatsu and Tamai reported the first successful finger replantation in 1968 [1]. Over the ensuing four decades, many refinements and advances had been made in microsurgical techniques resulting in a success rate of 92-99\% for digital replantation, but replantation surgery is still a challenge because the surgeon needs to perform the operation quickly while potentially be facing limited amount of tissue to work with $[2,3]$. Many factors can affect the prognosis, but impaired arteria digitalis communis is a particular problem for finger replantation surgery. Most surgeons treat digital amputation with damaged arteria digitalis communis as a complication of replantation. Both the proximal and distal neurovascular bundles are extensively damaged, making it difficult to

\footnotetext{
* Correspondence: cl_fly_214@163.com

'Department of Orthopedics, Shanghai Sixth People's Hospital East Affiliated to Shanghai University of Medicine \& Health Sciences, No. 222 Huanhu Xisan Road, Shanghai 201306, China

Full list of author information is available at the end of the article
}

estimate the level of tissue injury even with the help of an operating microscope. The aim of this study is to highlight the technical advantage of using cross-finger revascularization in salvage of digits with damaged arteria digitalis communis and the positive functional prognosis this can bring to the table.

\section{Materials and methods}

From July 2012 to June 2018, a retrospective study was conducted on 554 hands with total finger amputations treated by replantation of finger/fingers. Seven of these cases were complicated with damaged arteria digitalis communis and were replanted by anastomosing distal adjacent radial/ulnar digital artery to distal end of ulnar/ radial digital artery of amputation finger. Out of the 7 cases, 5 were males and 2 were females, with a mean age of 35.3 years old (ranging from 18 to 55 years old). All of them were work-related injuries. One case was complicated with a type III (according to Urbaniak's classification [4]) degloving injury in her right hand. All of the 7 
cases were followed up for a mean 19.1 months (ranging from 13 to 25 months). Assessments of hand functions were conducted at the end of this study; no other procedures were done before the assessment. One surgeon, who was not involved in the primary treatment of the patients, assessed the patients' hand functions, including total active motion, grip strength, and 2-point discrimination.

Each patient underwent radiographs of the hand(s), as well as blood routine examination, coagulation function, and electrocardiogram, right after being admitted to emergency room. No systemic diseases that could complicate the healing were reported.

Patients were told to remain in the supine position under brachial plexus anesthesia. The replantation team consists of two groups. One group brings the amputation part to a tagging table in the operation room once the patients arrive in the emergency room. The part is gently debrided with surgical preparation, and the blood vessels and nerves were examined by microsurgical instruments with the aid of operating microscope. Here, the surgeon will determine whether or not the part can be replanted, and whether vein grafting or cross-finger revascularization should be applied. The arteries and venous were tagged with small microvessel sutures. Next, the bone should be trimmed and shortened as needed, and two crossed Kirschner wires were retrograde inserted. Tendons were then traced through the tendon sheaths and tagged with tendon sutures.

Meanwhile, the proximal part could start being debrided by the other group once the anesthesia was set. The condition of the proximal vascular should be explored from distal to proximal in order to determine the degree of vascular injury, and it is the most important step in the systematic sequence according to individual surgeon's preference. Therefore, a second longitudinal incision was made at the mid-portion of the middle phalanx to the metacarpophalangeal (MP) joint of the adjacent digit, and the radial/ulnar digit artery was incised at the proximal interphalangeal joint (PIP joint). The arteries were tagged with microvessel clips. Then, the bony fixation and tendon repairs can be performed. The distal radial/ulnar digital artery of the adjacent finger was anastomosed to the distal stump of the ulnar/radial digital artery of the amputated finger. A skin pedicle was made by suturing the dorsal skin of the adjacent finger to the dorsal skin of the amputated finger and the palmar skin of the adjacent finger to the palmar skin of the amputated finger (Fig. 1). Nerve repair was performed if the nerve was not defect. Out of the 7 cases, 2 patients had nerve repaired.

Intraoperative intravenous dextran-40 was injected at the first anastomosis at $25 \mathrm{cc} / \mathrm{h}$. The dextran- 40 injection was continued for 5 days postoperatively. The

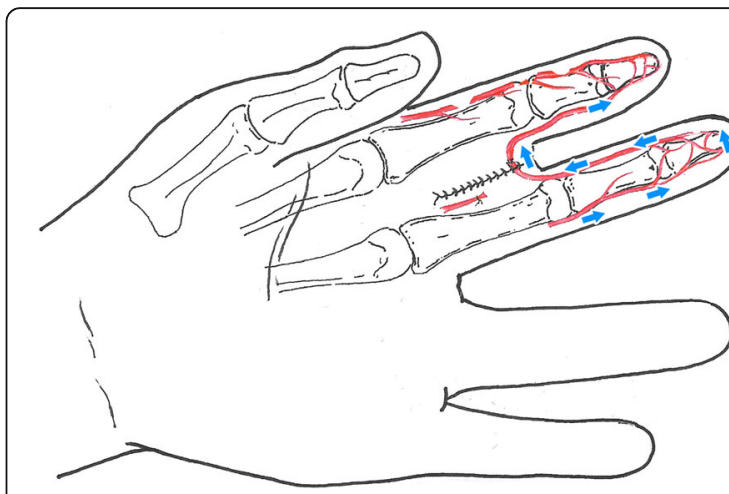

Fig. 1 A drawn figure shows the cross-finger revascularization technique and the direction of blood flow

anticoagulation protocols vary, depending on local practices. The hand and waist were elevated on pillows and kept warm. One hundred milligrams of oral acetylsalicylic acid was given for 30 days postoperatively.

All of the 7 cases were followed up for a mean 19.1 months (ranging from 13 to 25 months). Detachments of the amputated finger and adjacent finger were performed after 4 weeks and both fingers showed signs of good blood supply. The K-wires were removed simultaneously during the detachments. Passive functional exercises began right after detachments. Active functional exercise began 2 weeks later postoperatively.

\section{Results}

The mean follow-up period was 19.1 months (ranging from 13 to 25 months). As it is important of the vein treatment in the replantation, 3 to 4 veins were anastomosed in each patient. The total survival rate was $100 \%$. A total of 6 of patients (85.7\%) achieved protective sensation of replanted fingers. Mean 2-point discrimination was $8 \mathrm{~mm}$ static (ranging from 6 to $11 \mathrm{~mm}$ ), and mean grip strength was $35.3 \mathrm{~kg}$ (ranging from 29 to $40 \mathrm{~kg}$ ). Mean total active motion was $191.4^{\circ}$ (ranging from 170 to $\left.220^{\circ}\right)$. The lengths of the replanted digits compared with contralateral side ranged from 88 to 100\% (mean 93.3\%). No postoperative vascular complications occurred in our study.

\section{Case report}

A 38-year-old woman presented with a degloving injury in her right hand due to an industrial accident. Clinical and radiological examinations of her right hand showed PIP joint dislocation of the patient's index and middle fingers. The radial digital artery of her index finger was avulsed from the proximal phalanx, and the distal part of her index finger had no blood flow and was not revascularizable (Fig. 2).

After debridement and reduction of the dislocated PIP and MP joints, a long vein graft was taken from the 


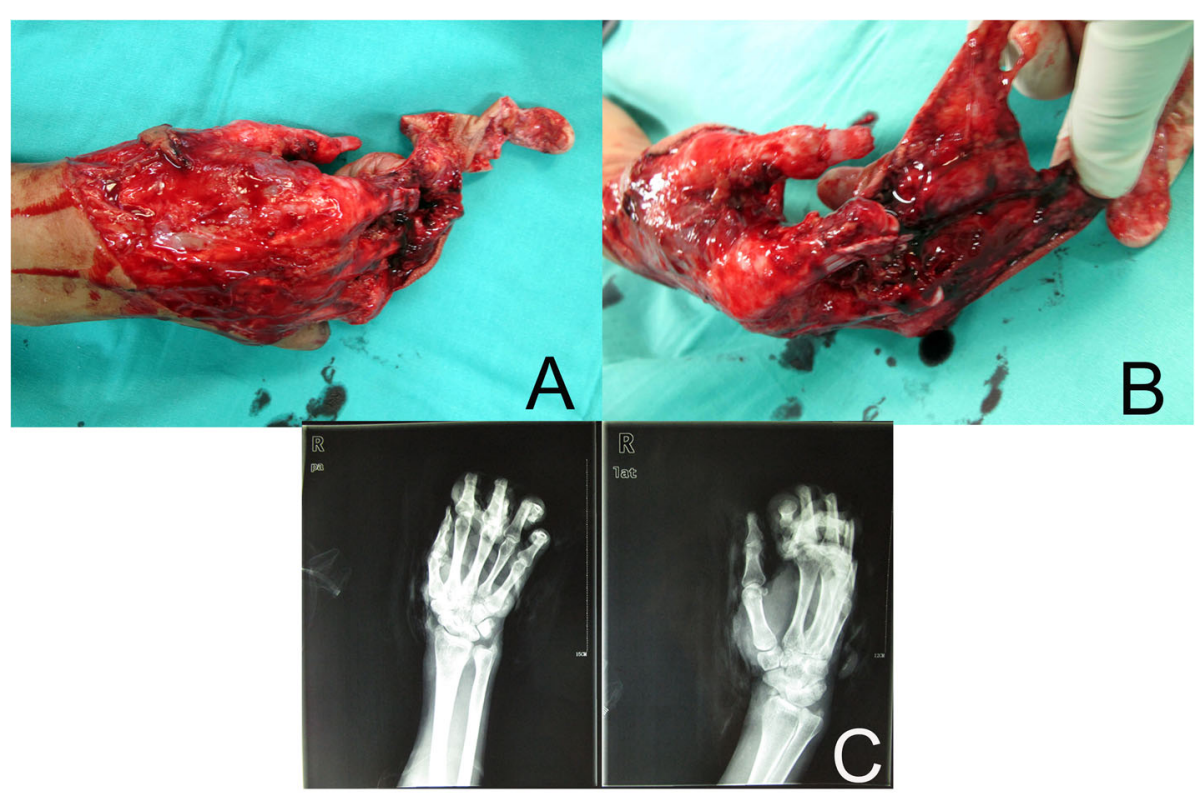

Fig. 2 Clinical and radiological examinations of her right hand. a, b Shows a degloving injury in her right hand with PIP joint dislocation of the patient's index and middle fingers. c Shows the radiological examination of her right hand

palmar side of her forearm to reconstruct an outflow course for her thumb and index finger. The degloving skin of her thumb and the dorsal surface of her hand were sutured in situ (Fig. 3a). The ulnar digital artery and nerve of her thumb were repaired to restore blood flow and sensation, respectively.

To restore blood supply to the patient's index finger, a mid-lateral skin incision was made from the distal interphalangeal joint (DIP joint) to the MP joint. The radial digital artery of the patient's index finger was severely injured and the proximal part could not be explored. The ulnar digital artery of her index finger was twisted. The incision was extended to the middle of her hand, as the first arteria digitalis communis was also injured. Therefore, a second incision was made at the midportion of the middle phalanx to the MP joint of the

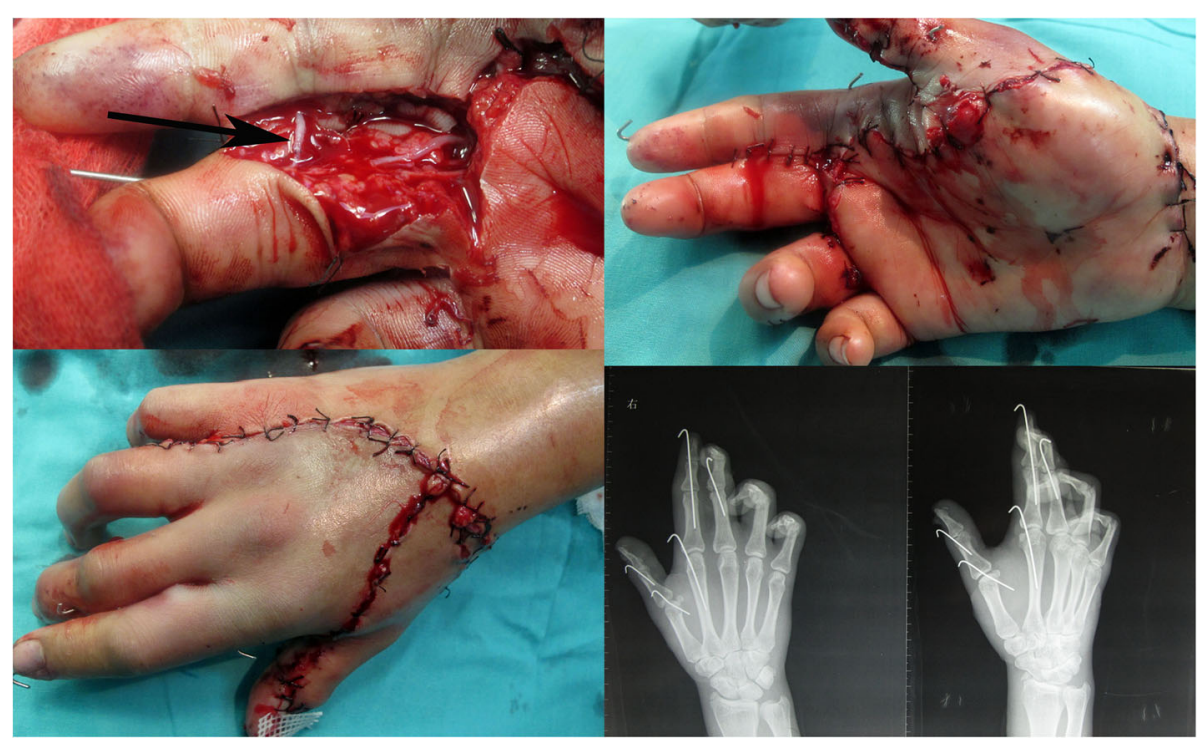

Fig. 3 The cross-finger revascularization technique for mangled finger salvage. a Shows the degloving skin of her thumb and the dorsal surface of her hand was sutured in situ. $\mathbf{b}$ Shows the distal radial digital artery of the middle finger was anastomosed to the distal stump of the ulnar digital artery of the index finger. c Shows a skin pedicle was made by suturing the dorsal skin of the middle finger to the palmar skin of the index finger 
middle finger, and the radial digital artery was incised at the PIP joint. The blood flow at the proximal stump of the radial digital artery was not ideal, which confirmed that the first arteria digitalis communis was damaged in the degloving injury. Since the blood flow from the distal radial digital artery of the middle finger was good, the distal radial digital artery of the middle finger was anastomosed to the distal stump of the ulnar digital artery of the index finger. A skin pedicle was made by suturing the dorsal skin of the middle finger to the palmar skin of the index finger. No circulatory problems occurred postoperatively (Fig. 3b, c).

Detachment of the index and middle fingers was performed 4 weeks after and both fingers showed signs of good blood supplies. The K-wires were also removed during the detachment. The appearance and function of the index and middle fingers were satisfactory 16 months postoperatively (Fig. 4).

\section{Discussion}

With the advent of microsurgery, digital and limb replantation has become a routine technique in most microsurgery centers worldwide. However, the survival rate of replanted digits is $45-65 \%$ in crushavulsion injuries, which is much lower compared to the more common amputations [5]. In avulsion injures, it is often difficult to estimate the degree of tissue injury, even with the help of an operating microscope $[5,6]$. Usually, proximal and distal neurovascular bundles are extensively damaged. After thorough debridement, widespread defects of the vascular structures can occur; thus, an end-to-end anastomosis is not suitable.
Many techniques have been applied to correct vascular defects, such as autogenous vein graft transfer and vessel rerouting from neighboring digits. Ozkan et al. used a long vein graft from the volar aspect of distal forearm to bridge the vessel defect in avulsion injuries [7]. The authors advised that careful attention should be paid to preventing any mismatch between the diameter of the vein graft and the vessels [7]. Reducing the length of the amputated proximal stump to compensate for the loss of vasculature is also an effective method. Among the patients in our study, both the radial and ulnar digital arteries of the amputated finger and the arteria digitalis communis were severely injured during the crush injury. However, most of the patients retained uninjured metacarpophalangeal joints or proximal interphalangeal joint, which would achieve good range of motion postoperatively as long as they are salvaged.

In these cases without any suitable vessels, the vein grafting, as backups for replantation attempt, it is difficult to provide sufficient arterial inflow to the traumatized bed [4, 8]. Furthermore, a large discrepancy in vessel size between the vein graft and the distal/proximal end of the artery is a great challenge to the surgeons. So, a syndactylia of the amputated and adjacent fingers was formed by suturing the dorsal skin of the adjacent finger to the dorsal skin of the amputated finger, and the palmar skin of the adjacent finger to the palmar skin of the amputated finger.

The most important functional prognosis is the preservation of finger sensibility. Based on the results of our study, a total of six patients achieved protective sensation, and mean 2-point discrimination was $8 \mathrm{~mm}$ static

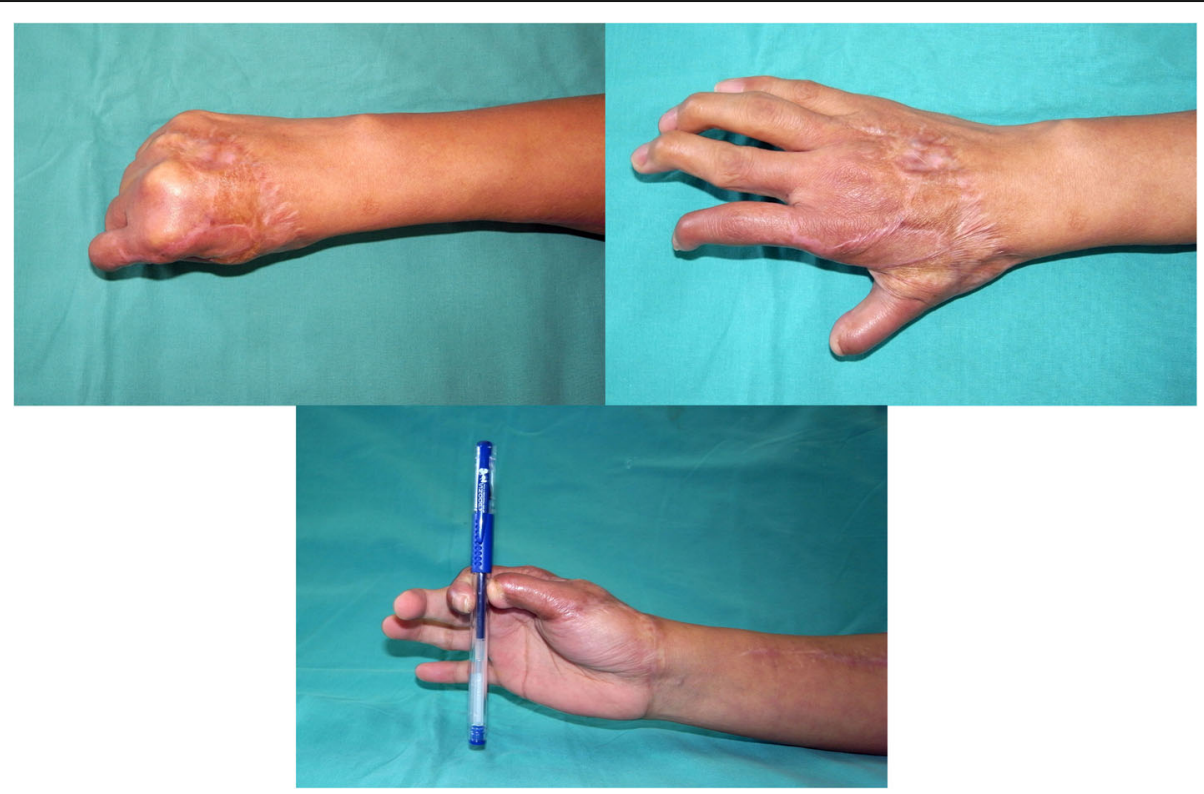

Fig. 4 The appearance and function of the index and middle fingers were satisfactory 
(ranging from 6 to $11 \mathrm{~mm}$ ). Although Ozcelik et al. observed an average of $7.2 \mathrm{~mm}$ static two-point discrimination in 31 replantations with no nerve repair in patients aged 6 to 40 years, and Faivre et al. reported an average of $4.6 \mathrm{~mm}$ static two-point discrimination in eight patients younger than 16 years with replantations distal to the distal interphalangeal joint $[9,10]$. The results in this study can possibly be explained by the greater degree of spontaneous neurotization in children. Most of the patients in our study are crush injuries, which can cause rupture and contusion of digital nerve. Thus, we performed nerve anastomosis on our patients when necessary.

Preservation of interphalangeal joint range of motion is also an important functional factor of finger replantation, especially since the period of immobilization could result in stiffness of the PIP and MP joints of both replanted and adjacent fingers. Based on our experience, with early range-of-motion exercises after K-wires removal, a good flexion-extension arc over $160^{\circ} \mathrm{can}$ be expected. Patients in our study have a mean active motion of $191.4^{\circ}$ (ranging from 170 to $220^{\circ}$ ) and mean grip strength of $35.3 \mathrm{~kg}$ (ranging from 29 to $40 \mathrm{~kg}$ ) through active strength exercises.

Another important thing to consider in this study is the patient with degloving injury. Vascular reconstruction of avulsed vessels is not an easy procedure. The survival rate of type III ring avulsion injuries treated by replantation has been reported to range from 73 to $85 \%$ [11]. Akuyrek et al. reported a series of 7 patients with type III ring avulsion injuries replanted by radical debridement and vein grafting [12]. As an attempt to increase the survival area of the re-attached skin, four veins on the dorsal and palmar sides were anastomosed respectively. Although the increased number of anastomosed vein might not be a significant indication of survival rate improvement, a large number of anastomosed veins may possibly improve the safety of the procedure if the quality of vessels walls is in question.

\section{Conclusion}

Although there are many other backup methods in the procedure of finger replantation, cross-finger revascularization technique is a good alternative option when both radial and ulnar arteria digitalis communis are badly injured and vein grafting cannot provide sufficient arterial inflow to the replanted finger. However, there are some limitations in this study, the reliability of the cross-finger revascularization technique needs to be further evaluated with a larger number of patients, and the function assessment should be included the adjacent digits in further clinical experiences. Nevertheless, with a comprehensive preoperative planning, this kind of injury has a high success rate, with good functional and aesthetic prognosis. On the other hand, good preoperative communication with the patient and the patients' family is still necessary.

\section{Abbreviations}

MR joint: Metacarpophalangeal joint; PIP joint: Proximal interphalangeal joint; DIP joint: Distal interphalangeal joint

\section{Acknowledgements}

We would like to thank all the staff in the Department of Orthopedic Surgery, Shanghai Sixth People's Hospital East Affiliated to Shanghai University of Medicine \& Health Sciences for their help on this study

\section{Authors' contributions}

LC and GW designed the study. SC wrote the first draft of the manuscript. LC and GW performed the procedure. SC collected the data. YC revised the manuscript. The author(s) read and approved the final manuscript.

\section{Funding}

This study is supported by grants from Shanghai Municipal Commission of Health and Family Planning Youth Project (grant number 20184Y0197).

\section{Availability of data and materials}

The datasets used and/or analyzed during the current study are available from the corresponding author on reasonable request.

\section{Ethics approval and consent to participate}

This study was approved by the Institutional Review Board of Shanghai Sixth People's Hospital East Affiliated to Shanghai University of Medicine \& Health Sciences. Written informed consent was obtained from all patients enrolled in the investigation.

\section{Consent for publication}

All the patients in this study have given their informed consent for the article to be published.

\section{Competing interests}

The authors declare that they have no competing interests.

\section{Author details}

${ }^{1}$ Department of Orthopedics, Shanghai Sixth People's Hospital East Affiliated to Shanghai University of Medicine \& Health Sciences, No. 222 Huanhu Xisan Road, Shanghai 201306, China. ${ }^{2}$ Department of Orthopedics, Shanghai Jiao Tong University Affiliated Sixth People's Hospital, 600 Yishan Road, Shanghai 200233, China

Received: 5 January 2020 Accepted: 4 March 2020

Published online: 11 March 2020

\section{References}

1. Komatsu S, Tamai S. Successful replantation of a completely cut-off thumb. Plastic Reconstructive Surg. 1968:42(4):374-7.

2. Chen SY, Wang CH, Fu JP, Chang SC, Chen SG. Composite grafting for traumatic fingertip amputation in adults: technique reinforcement and experience in 31 digits. The Journal of trauma. 2011;70(1):148-53.

3. Hahn HO, Jung SG. Results of replantation of amputated fingertips in 450 patients. J Reconstr Microsurg. 2006;22(6):407-13.

4. Urbaniak JR, Evans JP, Bright DS. Microvascular management of ring avulsion injuries. J Hand Surg (Am). 1981;6(1):25-30.

5. Mitchell GM, Morrison WA, Papadopoulos A, O'Brien BM. A study of the extent and pathology of experimental avulsion injury in rabbit arteries and veins. British journal of plastic surgery. 1985;38(2):278-87.

6. Kupfer DM, Eaton C, Swanson S, McCarter MK, Lee GW. Ring avulsion injuries: a biomechanical study. J Hand Surg (Am). 1999;24(6):1249-53.

7. Ozkan O, Ozgentas HE, Safak T, Dogan O. Unique superiority of microsurgical repair technique with its functional and aesthetic outcomes in ring avulsion injuries. Journal of plastic, reconstructive \& aesthetic surgery : JPRAS. 2006:59(5):451-9.

8. Tsai TM, Manstein C, DuBou R, Wolff TW, Kutz JE, Kleinert HE. Primary microsurgical repair of ring avulsion amputation injuries. J Hand Surg (Am). 1984;9A(1):68-72. 
9. Faivre S, Lim A, Dautel G, Duteille F, Merle M. Adjacent and spontaneous neurotization after distal digital replantation in children. Plastic Reconstructive Surg. 2003;111(1):159-65 discussion 66.

10. Ozcelik IB, Tuncer S, Purisa H, Sezer I, Mersa B, Kabakas F, et al. Sensory outcome of fingertip replantations without nerve repair. Microsurgery. 2008; 28(7):524-30.

11. Sanmartin M, Fernandes F, Lajoie AS, Gupta A. Analysis of prognostic factors in ring avulsion injuries. J Hand Surg (Am). 2004;29(6):1028-37.

12. Akyurek M, Safak T, Kecik A. Ring avulsion replantation by extended debridement of the avulsed digital artery and interposition with long venous grafts. Annals of plastic surgery. 2002;48(6):574-81.

\section{Publisher's Note}

Springer Nature remains neutral with regard to jurisdictional claims in published maps and institutional affiliations.

Ready to submit your research? Choose BMC and benefit from:

- fast, convenient online submission

- thorough peer review by experienced researchers in your field

- rapid publication on acceptance

- support for research data, including large and complex data types

- gold Open Access which fosters wider collaboration and increased citations

- maximum visibility for your research: over $100 \mathrm{M}$ website views per year

At BMC, research is always in progress.

Learn more biomedcentral.com/submissions 